\section{Red, amber and green: the role of the lung in de-priming active systemic neutrophils}

\author{
Elizabeth Sapey, ${ }^{1}$ Robert A Stockley ${ }^{2}$
}

There is little doubt that neutrophils, while vital in host defence, have the potential to cause significant tissue damage implicated both in lung and systemic diseases as well as systemic organ failure. Following pathogen recognition receptor activation by agents including formyl-methionyl-leucylphenylalanine (fMLP), cigarette smoke, cytokines or complement factor C5a, neutrophils are activated, recruited and participate in tissue breakdown and bacterial clearance via an array of mechanisms including phagocytosis, superoxide burst, extrusion of neutrophil extracellular traps (de-condensed chromatin and antimicrobial protein coated web-like structures), secretion of reactive oxygen and nitrogen species, and extrusion of a range of granule-derived proteases and antimicrobial peptides. These proteases, hydrolases and antimicrobial peptides are thought to contribute to many cellular functions including neutrophil migration, adherence and apoptosis, but their extra-cellular release is also associated with obligate tissue damage (as described in studies of quantum proteolysis ${ }^{1}$ ) and inflammation, and neutrophil proteinases in particular have been found to have a myriad of pro-inflammatory effects on bystander tissue to amplify inflammation, favouring further cell recruitment and degranulation.

In keeping with this, there are clear associations between neutrophils and many chronic inflammatory diseases as diverse as COPD, myocardial infarction, type 2 diabetes and inflammatory bowel disease. $^{2}$ Neutrophils have also been implicated widely in organ dysfunction, from acute kidney injury to acute lung injury, where neutrophil numbers correlate both with the severity of insult and negative outcomes. ${ }^{3}$

\footnotetext{
${ }^{1}$ Clinical and Experimental Medicine, The Medical School, University of Birmingham, Birmingham, UK; ${ }^{2}$ Department of Respiratory Medicine, University Hospital Birmingham NHS Foundation Trust, Birmingham, UK
}

Correspondence to Dr Elizabeth Sapey, Clinical and Experimental Medicine, The Medical School, University of Birmingham, Edgbaston, Birmingham B15 2TT, UK; lizsapey@aol.co.uk
Neutrophils exist in one of three states: quiescent, primed or active. Priming and activation appear distinct. In the primed state, there is no increase in oxidase activity; however, subsequent stimulation is able to provoke a response that is 10 -fold larger than in non-primed, activated cells. Individual primed cells are 'ready to go' but require further stimulation before activated responses are elicited. This is akin to a tri-colour warning system, with primed neutrophils held at 'amber', awaiting 'red' before activity is triggered. The mechanisms underlying priming are complex but require the generation of lipid second messengers generated by the phosphoinositide 3-hydroxykinase (PI3K) signalling system, especially PI3K $\gamma{ }^{4}$ This mechanism is pertinent, since recent studies have suggested constitutional PI3K activity as important in neutrophil immunosensecence ${ }^{5}$ and in chronic inflammatory diseases such as $\mathrm{COPD}^{6}$ which may support a hypothesis of perpetual priming in these conditions. Interestingly, these mechanisms may be different in animal models where neutrophils have been found to be less deformable. ${ }^{4}$

It is often considered that priming is an irreversible process, with amber only turning to red, but in health there is evidence that some priming is reversible (amber back to green). Studies using platelet activating factor (PAF) have demonstrated priming and de-priming by studying fMLP-stimulated superoxide anion generation, $\mathrm{CD} 1 \mathrm{lb}$ function, and cell polarisation and then subsequent repriming $2 \mathrm{~h}$ later with maintenance of full viability. ${ }^{7}$ This cyclical model of priming responses makes sense as it suggests a mechanism to limit unwarranted activation leading to self-harm caused by neutrophil degranulation. However, it was unclear whether de-priming occurred in vivo and, if so, where and how.

Neutrophil recruitment from blood to inflamed or infected tissue in the bronchial circulation has been described in detail. Red blood cells first induce margination of neutrophils towards the endothelium where they then undergo selectin-mediated rolling; this occurs primarily in the postcapillary venules (which are of $>15 \mu \mathrm{m}$ in diameter) because the shear force opposing neutrophil stopping is lower. P- and E-selectin are translocated to the apical endothelial surface where they engage glycosylated neutrophil ligands such as P-selectin glycoprotein ligand-1 and CD44. Chemoattractants derived from pathogen, tissue leucocytes or epithelial cells bind to cell surface receptors inducing an activating conformational change in neutrophilexpressed $\beta_{2}$ integrins allowing them to initiate firm neutrophil arrest and intravascular crawling. ${ }^{8}$ Diapedesis through the endothelial barrier then occurs, followed by a slower passage through the pericyte sheath and basement membrane.

While these processes describe neutrophil-endothelial interactions in the bronchial circulation (the 'classical mechanism' of rolling and tethering), there is evidence to suggest that a majority of neutrophils enter the lung from the pulmonary capillary network. Here neutrophil emigration is less well understood, but is thought to be largely independent of selectins and intracellular adhesion molecule-1 (ICAM-1). (and hence called the 'alternative mechanism's). Differences in mechanisms perhaps reflect the size constraints of the capillary networks as $40 \%-60 \%$ of these capillaries are narrower than a spherical neutrophil. ${ }^{9} 10$

To pass through these small structures, a neutrophil must change its shape, and models suggest that while almost $100 \%$ of 'rolling' neutrophils remain spherical, neutrophils passing through capillary beds elongate in shape.$^{112}$ The distinct multilobulated nucleus of neutrophils and nuclear envelope composition, particularly levels of lamins $\mathrm{A} / \mathrm{C}$, are thought crucial to cell passage through a narrow pore, as individual lobes sequentially 'thread' through constrictions. ${ }^{13}$ Close contact with the capillary wall is thought to cause transient deformation, ${ }^{14-16}$ leading to small regional differences in cell rheology with more stiffening of the central body compared with the leading edge of the cell. ${ }^{17}$ This process is considered to occur in the absence of priming or activation.

Priming is crucial to diapedesis from blood to tissues as it leads to cytoskeletal rearrangement, generating cell polarity. Neutrophils extend filamentous actin rich structures (pseudopods) at the leading edge while simultaneously cross-linking actin, releasing points of adhesion and contracting non-muscle myosin II at the rear of the cell to help manipulate the plasma membrane in the correct direction. Priming and cytoskeletal rearrangement render the neutrophil more stiff, 


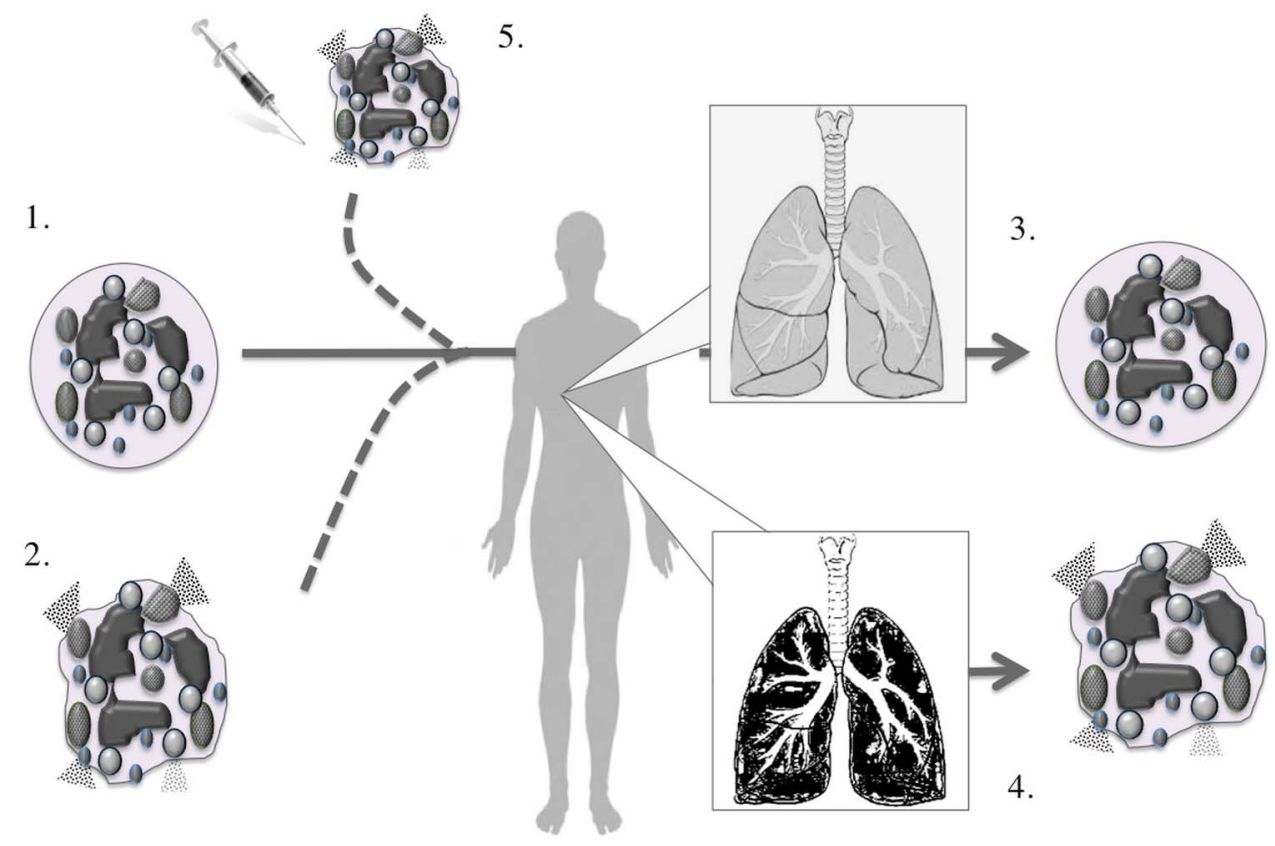

Figure 1 Factors that may influence neutrophil transit time through the pulmonary circulation. In health, there may be no delay and no significant retention of neutrophils in the pulmonary circulation $(1,3)$. Primed neutrophils (caused by systemic or pulmonary inflammation or infection) will be retained in the pulmonary circulation; (2) however, some priming is reversible and once de-primed by the pulmonary endothelium, quiescent cells will be released back into the circulating blood. (3) In inflammatory diseases of the lungs, this de-priming mechanism fails, leading to the release of primed cells (4) that may also have potential systemic inflammatory consequences. In experimental models, methods of neutrophil isolation or labelling may cause priming, leading to neutrophil retention within the pulmonary capillaries (5).

hindering its passage through the pulmonary circulation and favouring retention and diapedesis into the lung tissue.

It was thought that the conformational changes required to allow neutrophils through the pulmonary capillaries caused a delay in neutrophil transit through the lung, ranging from a few seconds to more than 20 min $^{18}$ with approximately $50 \%$ of neutrophils stopping in their passage through the lung. ${ }^{19}$ This was in contrast to red blood cells, which generally traverse the lung in a few seconds with little or no observable delay. ${ }^{21}$ It was proposed that this led to retention of neutrophils in the pulmonary circulation at concentrations of 35-100 times greater than in large vessels of the systemic circulation. ${ }^{12} 2223$ It was further hypothesised that this was a physiological state leading to the presence of a marginated pool of leucocytes in the lungs, which might be vital for host defence. ${ }^{19}$

These initial studies were conducted in animals and although a human study did not reproduce this slower transit time, ${ }^{24}$ it was concluded that this anomalous result represented inadequacies in $\gamma$ camera sensitivity at the time. Slower neutrophil transit times in the pulmonary circulation were heightened in disease, allowing significant retention and transmigration into the lung. ${ }^{25}$ MacNee and coworkers demonstrated that cigarette smoke exposure and inflammation led to increased retention in the pulmonary circulation and considered this to be important in tissue destruction leading to the development of emphysema. ${ }^{24} 26$

However, a major problem in the study of isolated neutrophils is the inadvertent priming of the cells by the purification process itself. Studies of PMN function in vivo pose particular technical problems especially if the cells have to be isolated and labelled. Theoretically, any practice that primes neutrophils will render them more stiff, hinder their passage through the capillary network, slow their transit time and favour retention in the lung. Recent studies have recognised the importance of careful isolation of neutrophils and labelling to reduce inadvertent priming that would alter the interpretation of subsequent results when cells are re-infused for study. There are many factors that might influence a neutrophil's journey through the pulmonary capillaries that must be considered: the model (animal vs human), the study population (patients vs controls) and the techniques used to study this are described in figure 1.

The current paper $^{27}$ examines this phenomenon in careful detail and provides three fascinating observations. First, in health, quiescent neutrophils have a short transit time in the lung with only minimal delay and retention. Second, priming leads to retention of the vast majority of cells, but that the priming agent (PAF vs granulocyte macrophage colony-stimulating factor (GM-CSF)) may in part determine whether this is an irreversible or reversible process (as previously described $^{7}$ ). Third, the lung can de-prime neutrophils, and hence play a modulatory role in neutrophil response locally and systemically, which can be lost in inflammatory lung disease.

The implications are manifold. First, it brings into question what we thought we knew about the dynamics of neutrophil passage through the lungs. It highlights a methodology that enhances our understanding of the role of the neutrophil in pathophysiology in real time. It also suggests a new therapeutic avenue in inflammatory lung disease where restoring the ability of the lung to de-prime activated neutrophils (changing amber to green) may improve outcomes.

Contributors ES wrote the article with input from RAS.

Funding Medical Research Council, MR/L008335/1. Competing interests None. 
Provenance and peer review Commissioned; internally peer reviewed.

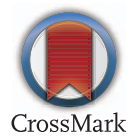

To cite Sapey E, Stockley RA. Thorax 2014;69:606-608.

Thorax 2014;69:606-608.

doi:10.1136/thoraxjnl-2014-205438

\section{REFERENCES}

1 Liou TG, Campbell EJ. Quantum proteolysis resulting from release of single granules by human neutrophils: a novel, nonoxidative mechanism of extracellular proteolytic activity. J Immunol 1996;157:2624-31.

2 Caielli S, Banchereau J, Pascual V. Neutrophils come of age in chronic inflammation. Curr Opin Immunol 2012;24:671-7.

3 Grommes J, Soehnlein 0. Contribution of neutrophils to acute lung injury. Mol Med 2011:17:293-307.

4 Condliffe AM, Davidson $\mathrm{K}$, Anderson $\mathrm{KE}$, et al. Sequential activation of class IB and class IA PI3K is important for the primed respiratory burst of human but not murine neutrophils. Blood 2005; 106:1432-40.

5 Sapey E, Greenwood H, Walton GM, et al. Phosphoinositide 3 kinase inhibition restores neutrophil accuracy in the elderly: towards targeted treatments for immunesenescence. Blood 2014;23:239-48.

6 Sapey E, Stockley JA, Greenwood H, et al. Behavioral and structural differences in migrating peripheral neutrophils from patients with chronic obstructive pulmonary disease. Am J Respir Crit Care Med 2011;183:1176-86.
7 Kitchen E, Rossi AG, Condliffe AM, et al. Demonstration of reversible priming of human neutrophils using platelet-activating factor. Blood 1996:88:4330-7.

8 Wagner JG, Roth RA. Neutrophil migration mechanisms with an emphasis on pulmonary vasculature. Pharmacol Rev 2000;52:349-74.

9 Schmid-Schonbein GW, Shih YY, Chien S.

Morphometry of human leukocytes. Blood 1980;56:866-75.

10 Schmid-Schonbein GW, Skalak R, Usami S, et al. Cell distribution in capillary networks. Microvasc Res 1980;19:18-44.

11 Gebb SA, Graham JA, Hanger CC, et al. Sites of leukocyte sequestration in the pulmonary microcirculation. J Appl Physiol 1995;79:493-7.

12 Doerschuk CM, Beyers N, Coxson HO, et al. Comparison of neutrophil and capillary diameters and their relationship to neutrophil sequestration in the lung. J App/ Physiol 1993;74:3040-5.

13 Rowat $A C$, Jaalouk $D E$, Zwerger $M$, et al. Nuclear envelope composition determines the ability of neutrophil-type cells to passage through micron-scale constrictions. J Biol Chem 2013;288:8610-18.

14 Kadash KE, Lawrence MB, Diamond SL. Neutrophi string formation: hydrodynamic thresholding and cellular deformation during cell collisions. Biophys $J$ 2004:86:4030-9.

15 Zhou C, Yue P, Feng J. Simulation of neutrophil deformation and transport in capillaries using Newtonian and Viscoelastic drop models. Ann Biomed Eng 2007:35:766-80.

16 Le Roux M, Magnaudet J. Simulation of neutrophil motion and deformation: influence of rheology and flow configuration. Comput Methods Biomech Biomed Engin 2011;14(Supp 1):107-9.

17 Pai A, Sundd P, Tees DFJ. In situ microrheological determination of neutrophil stiffening following adhesion in a model capillary. Ann Biomed Eng 2008;36:596-603.

18 Lien DC, Wagner WW, Capen RL, et al. Physiologica neutrophil sequestration in the lung: visual evidence for localization in capillaries. J App/ Physiol 1987;62:1236-43.

19 Hogg JC. Neutrophil kinetics and lung injury. Physiol Rev 1987:67:1295-8.

20 Huang Y, Doerschuk CM, Kamm RD. Computational modeling of RBC and neutrophil transit through the pulmonary capillaries. J App/ Physiol 2001;90: 545-64

21 Presson RG, Graham JA, Hanger CC, et al. Distribution of pulmonary capillary red blood cell transit times. J App/ Physiol 1995;79: 382-8

22 Gee MH, Albertine KH. Neutrophil-endothelial cell interactions in the lung. Annu Rev Physiol 1993:55:227-48.

23 Doershuk CM, Allard MF, Martin BA, et al. The marginated pool of neutrophils in the lung of rabbits. J Appl Physiol 1987;63:1806-16.

24 MacNee W, Wiggs B, Belzberg AS, et al. The effect of cigarette smoking on neutrophil kinetics in human lungs. $N$ Engl J Med 1989;321: 924-8.

25 Yoshida K, Kondo R, Wang Q, et al. Neutrophil cytoskeletal rearrangements during capillary sequestration in bacterial pneumonia in rats. Am $\mathrm{J}$ Respir Crit Care Med 2006;174:689-98.

26 Brown GM, Brown DM, Donaldson K, et al. Neutrophil sequestration in rate lung. Thorax 1995;50: 661-7.

27 Summers C, Singh NR, White JF, et al. Pulmonary retention of primed neutrophils: a novel protective host response, which is impaired in the acute respiratory distress syndrome. Thorax 2014:69:623-9. 